\title{
AN APLANATION TONOMETER OF NEW PRINCIPLE*
}

\author{
BY \\ D. M. MAURICE \\ From the Ophthalmological Research Unit, Medical Research Council, \\ Institute of Ophthalmology, University of London
}

THE impression type of tonometer is probably sufficiently accurate for most clinical purposes, and for some scientific investigations where the changes in individual eyes are under observation. For the accurate measurement of the intra-ocular pressure it is, however, inadequate, since its reading is a function both of this and of the distensibility of the tunic of the eye. Friedenwald (1937), in his full and careful investigation of this question, has devised a method which compensates to some extent for the variations in this distensibility, but at the cost of increasing the uncertainties of measurement resulting from the poor sensitivity of the instrument. Aplanatic tonometers of the Maklakow type, which at first sight seem to provide an absolute measure of the intra-ocular pressure, also give readings influenced by the distensibility of the globe, but even if allowance be made for this there remain large errors due to factors not included in the simple theoretical treatment (Friedenwald, 1939; Apin, 1928). For many problems in intra-ocular fluid dynamics a more sensitive instrument is required, and one which will give a true measure of the intra-ocular pressure; the tonometer described here was designed to meet this need, and is found in practice to go a long way towards giving satisfaction.

\section{PRINCIPLE}

If two inflated membranes are pressed together, their surface of contact will be plane if the pressures within them are equal, regardless of the initial radii of curvature of the membranes or of the nature of their contents as long as they are fluid. If the surface between such a membrane and the cornea can then be made flat, the pressure within the mémbrane is equal to the intra-ocular pressure, with the addition of a small correction for the rigidity of the cornea. The adjustment of the pressure can be most conveniently controlled by observing the reflection of some object in the interface and comparing it with that in an adjacent plane surface.

\section{CONSTRUCtion}

The practical construction of the instrument in its present experimental stage of development is shown in Figs 1 and 2. A cylindrical chamber, $1 \mathrm{~cm}$. in diameter, is

- Received for publication November 13, 1950. 
turned out of perspex or metal, fitted with a connecting tube at the side, and sealed at one end with a transparent wall set at an angle to avoid confusing reflections. Near
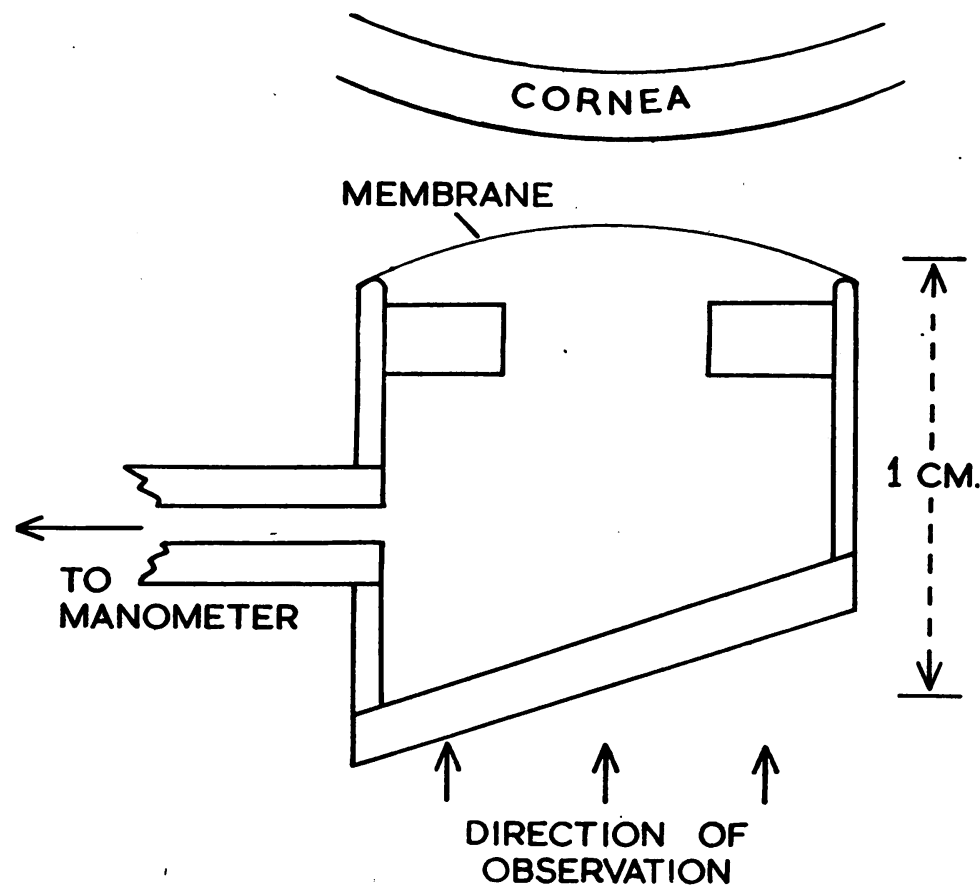

FIG. 1.-Cross section of chamber.

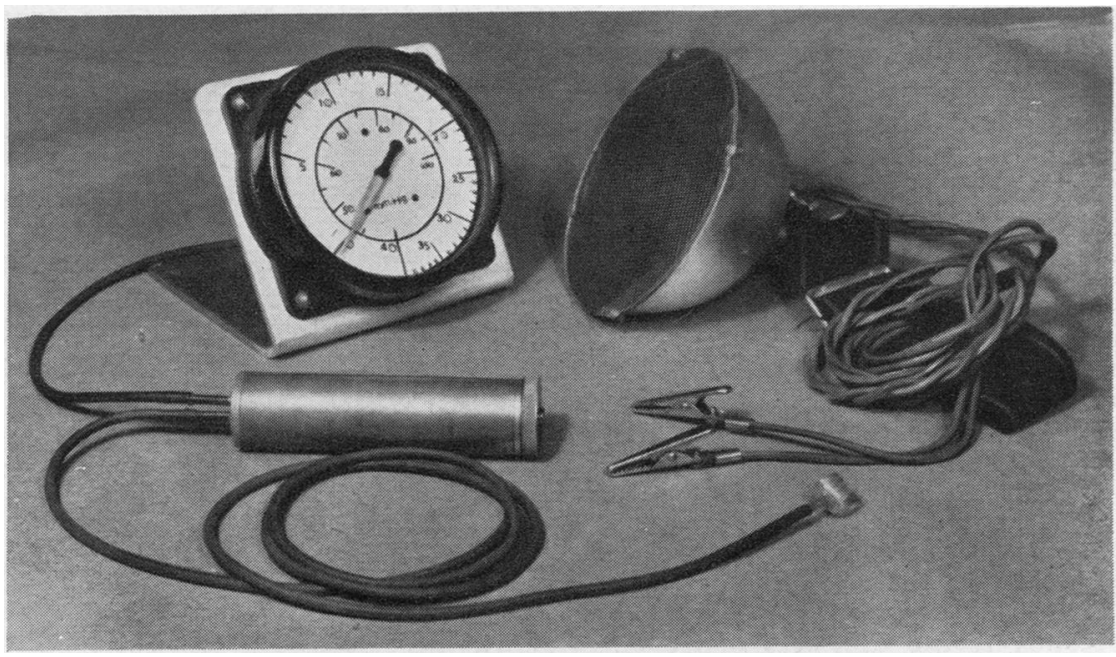

Fig. 2.-Complete apparatus. 
the other end of the chamber is fixed a ring of perspex to act as the comparison plane reflecting surface, and in the centre of this a $4-\mathrm{mm}$. hole is drilled to allow observation of the image in the interface between the cornea and membrane. The membrane which closes this end of the chamber is made from "Pliofilm ", a plasticized rubber sheet which has the necessary properties of toughness, flexibility, specular reflection, and elasticity. This last is important in order that the membrane can assume a spherical shape under pressure, and will not pucker when the interface becomes plane. In addition, it has the advantages that it is impermeable to water so that misting over of the internal reflecting surfaces is avoided, and that it is thermoplastic so that it can be easily fixed in position without distortion. To achieve this the pliofilm is stretched over a metal ring as in a tambourine and warmed over a flame until soft. The front of the chamber is painted with a special adhesive, and the membrane is drawn over this, pressed down on to its sides, cut free, and trimmed.

The object observed by reflection is a system of parallel straight opaque lines, $1 \mathrm{~mm}$. wide and spaced $1 \mathrm{~mm}$. apart, in the form of a photographic negative, which is mounted on the observer's forehead over his dominant eye. This negative is illuminated from behind by a bulb in a diffusing reflector, the current coming from a battery in his pocket.

The chamber is connected by thin rubber tubing to a device for adjusting the pressure and to an aneroid manometer for recording it. These would preferably be in one piece so that the entire apparatus could be held in the observer's hands.

\section{Practical Application}

A local anaesthetic is instilled into the eye and also a drop of paroleine to prevent adhesion between the membrane and corneal epithelium. If this precaution is taken the instrument appears to be completely non-traumatic; readings were taken continuously on one eye for a total of hour over a 1 ! hour period without any subsequent ill effects. The pressure in the chamber is now raised to a value a little below that expected in the eye. With the room in semi-darkness, the front of the chamber is approximated to the cornea, so that the system of lines can be seen reflected in the perspex ring, and their small corneal image is visible through the central hole. Contact between the membrane and cornea is now made, and sufficient pressure is applied so that the interface just fills the central hole. With a little practice these operations present no difficulty. If the pressure in the chamber is below the intra-ocular pressure, the interface between membrane and cornea will be convex towards the observer and hence the lines imaged in the centre will be smaller and more crowded together than those in the outer plane perspex ring (Fig. $3 b$ ). As the pressure is raised the central lines will expand until, when the interface is plane, they become the same size as those in the outer ring, and accordingly this is the correct setting of the instrument (Fig. 3a). One set of lines is not necessarily parallel with the other as this condition is dependent on how the interface is oriented. As the pressure in the tonometer is raised above the correct setting, the central lines become larger (Fig. 3c), then too large for observation, and finally smaller again as the interface becomes more concave towards the observer. The image in the centre will appear as sharp and bright as that in the polished perspex if the conditions are correct, but any particles of dirt or fine hairs between the membrane and cornea are sufficient to cause distortion 
and must be wiped or washed away. If the distortion is very serious and uncontrollable, owing, for example, to a poor epithelial surface, a fairly accurate estimate of the point at which the interface becomes plane can

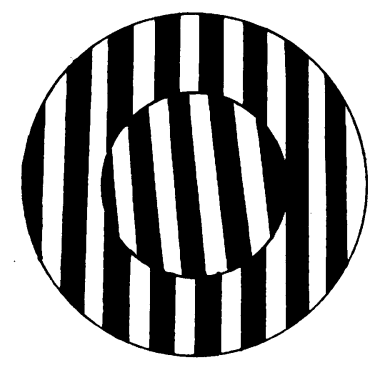

(a)

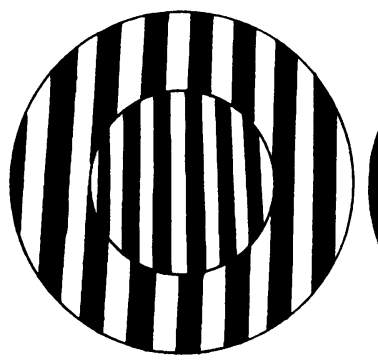

(b)

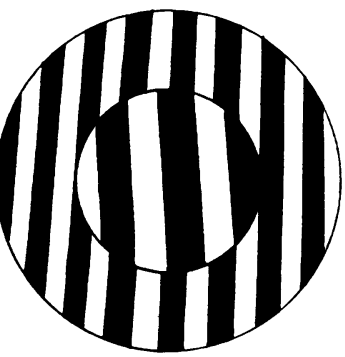

(c)

FIG. 3.-Appearance of reflections under various conditions of adjustment: outer ring, perspex plane comparison surface; centre, membrane/cornea interface. (a) Adjustment correct. (b) Tonometer pressure too low.

(c) Tonometer pressure too high.

be made, with experience, by observing the distribution of light over it and how this changes with the movements of the observer's head.

As the instrument has a very rapid reaction time, the image in the normal eye fluctuates in size with the pulse and respiratory rhythm of the intra-ocular pressure. The measurement of these variations, about $2.5 \mathrm{~mm}$. Hg each in one subject, might be valuable for some purposes, but they delay and render more difficult the estimation of the average intraocular pressure which is more generally required. For this reason experiments are now being directed to slowing the response of the instrument by filling the chamber with a viscous oil, and to the maintenance at the same time of an adequate brightness in the reflected images.

SENSITIVITY.-Ignoring the effects of corneal rigidity the curvature of the interface $(\mathrm{K})$ is readily shown to be connected with the pressures and radii of curvature corresponding to the tonometer $\left(p_{t}\right.$ and $\left.r_{t}\right)$ and to the eye $\left(p_{e}\right.$ and $\left.r_{e}\right)$ by the formula:

$$
\mathrm{K}=\frac{p_{t}-p_{e}}{r_{t} p_{t}+r_{e} p_{e}}
$$

Hence the sensitivity is given by:

$$
\frac{d \mathrm{~K}}{d p_{e}}=\frac{1}{\left(r_{t}+r_{e}\right) p_{e}} \text { at the correct setting } p_{t}=p_{e} .
$$

The sensitivity is therefore reduced inversely with $r_{t}$ when this is large compared with $r_{e}$, and maximum sensitivity is obtained by softening the membrane by gentle heat before use so that it is readily given a high curvature by a low pressure; it will remain sufficiently soft for several hours after once being warmed. With $r_{t} \bumpeq r_{e}$ a change of 2 per cent. in the pressure from equality should result in the formation of a mirror of curvature of just over one dioptre, which should be readily distinguish- 
able from a plane surface. In eyes of experimental animals where the anterior chamber is in sufficiently free connection with a manometer to abolish variations of pulse and respiration, differences of pressure of this order can be distinguished in practice.

ABSOLUTE ACCURACY.- In such cannulated eyes in experimental animals the influence of the rigidity of the cornea can be assessed, particularly at low pressures where the sensitivity is high, in terms of absolute pressure differences. The tonometer in the case of the cat's eye is found to give a reading $1.5 \mathrm{~cm}$. water too high, and in that of the rabbit $1 \mathrm{~cm}$. too high. These differences appear to be constant at different pressures and at the highest tested, $60 \mathrm{~mm}$. $\mathrm{Hg}$, the tonometric and manometric pressures agree within the limits of error. The thickness of the human cornea is intermediate between that of the cat and rabbit, and it is reasonable to suppose that the error it causes is also intermediate, that is, about $1 \mathrm{~mm} . \mathrm{Hg}$.

The influence of the distensibility of the globe can be estimated by using the nomograms given in Friedenwald's papers; the " rigidity coefficient" may be taken to vary between the extremes of 0.03 and 0.01 , his results suggesting that these represent the range for normal eyes. With this tonometer giving a reading of $30 \mathrm{~mm}$. $\mathrm{Hg}$, the true range of intra-ocular pressure would be 27 to $29 \mathrm{~mm}$. $\mathrm{Hg}$, and if the diameter of contact is brought down to $3 \mathrm{~mm}$. the error is negligible. By comparison, the Schiotz tonometer, with a weight of $10 \mathrm{~g}$. and a scale reading of 10 , would represent a true range of intra-ocular pressure of 18 to $34 \mathrm{~mm}$. $\mathrm{Hg}$, and the Fick-Livschitz aplanation tonometer, with the standard contact diameter of $6.8 \mathrm{~mm}$. and reading $30 \mathrm{~mm}$. Hg, a true range of 12 to $22 \mathrm{~mm}$. $\mathrm{Hg}$. In addition, according to Friedenwald, aplanatic instruments of this type show a systematic error of about $10 \mathrm{~mm}$. $\mathrm{Hg}$.

\section{SUMMARY}

(1) A description is given of a tonometer operating on the principle that a plane surface will be established between the cornea and an inflated membrane pressed against it when the pressure inside the membrane is equal to the intra-ocular pressure.

(2) It has the major advantages of giving absolute readings to within an accuracy of $1 \mathrm{~mm}$. $\mathrm{Hg}$, and of having a sensitivity of about 2 per cent. of the intra-ocular pressure.

(3) It has the disadvantage of a fast reaction time so that pulse and respiratory changes in the intra-ocular pressure delay the determination of its mean value.

My thanks are due to the Goodyear Tyre and Rubber Co. (Great Britain) Ltd. of Wolverhampton, and to National Adhesives, Ltd. of Slough, for supplying me with samples of "Pliofilm" and adhesive respectively, and to Mr. C. Purvis for constructing the apparatus.

\section{REFERENCES}

ApIN, K. (1928). Klin. Mbl. Augenheilk., 81, 631.

Friedenwald, J. S. (1937). Amer. J. Ophthal., 20, 985. (1939). Ibid., 22, 375 